\title{
Chronic thromboembolic pulmonary hypertension in pediatric patients
}

\author{
Michael M. Madani, MD, ${ }^{\mathrm{a}}$ Lara M. Wittine, MD, ${ }^{\mathrm{b}}$ William R. Auger, MD, ${ }^{\mathrm{b}}$ Peter F. Fedullo, MD,

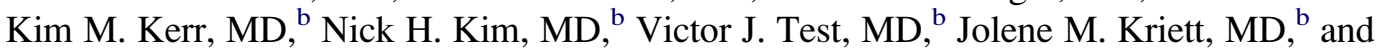 \\ Stuart W. Jamieson, MB, FRCS ${ }^{\mathrm{a}}$
}

\begin{abstract}
Objective: Chronic thromboembolic pulmonary hypertension is a rare form of pulmonary hypertension that can lead to progressive right heart failure and death. Pulmonary thromboendarterectomy surgery is the treatment of choice resulting in significant improvements in functional status, cardiopulmonary hemodynamics, and survival. This study reports the largest case series of pediatric patients with chronic thromboembolic pulmonary hypertension who underwent pulmonary thromboendarterectomy surgery at one institution.
\end{abstract}

Patient and Methods: The University of California, San Diego, chronic thromboembolic pulmonary hypertension database identified patients 18 years or younger at the time of pulmonary thromboendarterectomy surgery $(\mathrm{n}=17)$. Medical charts were reviewed for hemodynamics, thromboembolic risk factors, and postoperative outcomes.

Results: Pulmonary thromboendarterectomy surgery in pediatric patients resulted in improved functional status and significantly improved cardiopulmonary hemodynamics: mean arterial pressure decreased from $45.5 \mathrm{~mm}$ $\mathrm{Hg} \pm 20.7$ to $27.3 \pm 13.0 \mathrm{~mm} \mathrm{Hg}(P=.00073)$, pulmonary vascular resistance decreased from $929 \pm$ dynes $\cdot \mathrm{s} \cdot \mathrm{cm}^{-5}$ to $299 \pm 307$ dynes $\cdot \mathrm{s} \cdot \mathrm{cm}^{-5}(P=.0012)$, and cardiac output improved from $3.8 \pm 1.1 \mathrm{~L} / \mathrm{min}$ to $5.6 \pm 1.6 \mathrm{~L} / \mathrm{min}(P=.0061)$. There were no deaths during surgery or 30 days after surgery, and long-term survival (5+years) was achieved in $87.5 \%$. As compared to adults with chronic thromboembolic pulmonary hypertension, there was a higher rate of rethrombosis in pediatric patients $(38 \%$ vs $1 \%-4 \%)$.

Conclusions: This study demonstrates that pulmonary thromboendarterectomy surgery in pediatric patients with chronic thromboembolic pulmonary hypertension is well tolerated with improved postoperative hemodynamics, functional status, minimal postoperative complications, and low perioperative mortality, similar to that reported for adults with chronic thromboembolic pulmonary hypertension, with the notable exception being a higher rate of rethrombosis in pediatric patients. (J Thorac Cardiovasc Surg 2011;141:624-30)

Chronic thromboembolic pulmonary hypertension (CTEPH) is an uncommon, yet potentially curable form of pulmonary hypertension. ${ }^{1}$ It occurs in $0.5 \%$ to $3.8 \%$ of patients after acute or recurrent pulmonary embolus (PE) when there is incomplete resolution of the thrombus burden., ${ }^{2,3}$ Transformation to a fibrotic residua results in chronic proximal vessel obstruction. This, along with the development of a distal arteriopathy similar to that seen in idiopathic pulmonary arterial hypertension, ${ }^{4}$ can lead to pulmonary hypertension and right ventricular overload. If left untreated, right heart failure and death are the inevitable end results. ${ }^{5}$ Pulmonary thromboendarterectomy (PTE) surgery, however, offers an effective treatment for this condition,

From the Department of Cardiothoracic Surgery ${ }^{\mathrm{a}}$ and the Division of Pulmonary and Critical Care Medicine, ${ }^{\mathrm{b}}$ University of California San Diego, San Diego, Calif.

Disclosures: Authors have nothing to disclose with regard to commercial support.

Received for publication March 13, 2010; revisions received June 17, 2010; accepted

for publication July 5, 2010; available ahead of print Aug 30, 2010.

Address for reprints: Michael M. Madani, Department of Cardiothoracic Surgery,

University of California, San Diego, 200 West Arbor Drive, MC 8892, San Diego,

Calif; 92103-8892 (E-mail: mmadani@ucsd.edu).

$0022-5223 / \$ 36.00$

Copyright (c) 2011 by The American Association for Thoracic Surgery doi: $10.1016 /$ j.jtcvs.2010.07.010 significantly improving cardiopulmonary hemodynamics, gas exchange, functional status, and survival. ${ }^{6,7}$

As venous thromboembolism is increasingly recognized in the pediatric patient population, ${ }^{8}$ CTEPH should be an important diagnostic consideration in symptomatic children with a known hypercoagulable state, a history of thromboembolism or venous catheter placement, and/or a diagnosis of pulmonary hypertension. ${ }^{9,10}$ This study presents a case series of pediatric patients ( $>18$ years of age) with CTEPH who underwent PTE surgery at our institution. The demographics, risk factors, surgical outcomes, and postoperative complications in this unique subgroup of CTEPH patients are reported.

\section{PATIENTS AND METHODS}

Retrospective review of the University of California San Diego's (UCSD) CTEPH database identified patients who were 18 years of age or younger at the time of their PTE surgery. For the purposes of this study, informed consent was waived and data collected in concordance with the standards set by our institutional review board. All patients have been referred for consideration of surgery, and nearly all underwent a standard preoperative evaluation including chest radiograph, transthoracic echocardiography, ventilationperfusion scan, pulmonary angiography, right heart catheterization, and hypercoagulable workup before or during their consultation at UCSD. 


\section{Abbreviations and Acronyms \\ $\mathrm{CTEPH}=$ chronic thromboembolic pulmonary hypertension \\ ICU = intensive care unit \\ INR = international normalized ratio \\ $\mathrm{PE} \quad=$ pulmonary embolus \\ PTE = pulmonary thromboendarterectomy \\ PVR = pulmonary vascular resistance \\ UCSD = University of California San Diego \\ WHO $=$ World Health Organization}

Patients were operated on by 1 of 3 surgeons between 1998 and 2009 using a previously described standard technique. ${ }^{11}$ PTE involved a median sternotomy, cardiopulmonary bypass, and deep hypothermia with periods of circulatory arrest, followed by consecutive endarterectomy of the right and left pulmonary arteries. This resulted in the removal of organized thromboembolic material from the vessel intima that was classified according to the Jamieson scoring system as type 1 disease (semiorganized and chronic thrombus in main or lobar pulmonary arteries), type 2 (fibrotic residua in lobar or proximal segmental arteries without visible thrombus), type 3 (distal segmental disease without visible thrombus), or type 4 (inoperable disease/ distal arteriolar vasculopathy). ${ }^{11}$ During the rewarming period, a persistent foramen ovale (if present) was surgically closed so long as pulmonary pressures had sufficiently normalized.

Patients' immediate postoperative care took place in the intensive care unit (ICU), where mechanical ventilation, inotropic support, and aggressive diuresis were provided as long as necessary for recovery. For those patients believed to be at a higher risk for rethrombosis after endarterectomy, such as those with antiphospholipid syndrome or a main pulmonary artery endarterectomy, intravenous heparin was started within 6 to 12 hours after the operation, once chest tube drainage had significantly abated. In those without these risk factors, subcutaneous heparin was initiated. Postoperative thrombosis prophylaxis continued until the resumption of warfarin anticoagulation resulted in a therapeutic international normalized ratio (INR). Postoperative cardiopulmonary hemodynamics were measured by thermodilution catheterization techniques before transfer out of the ICU with the patients off both mechanical ventilation and inotropic agents. All other postoperative studies, including transthoracic echocardiogram, ventilation-perfusion scan, rest and exercise oxygen saturation testing, and chest radiography occurred while the patients were convalescing on the general hospital ward. Long-term follow-up (3 months to 7 years) was performed by referring physicians, who communicated pertinent findings (echocardiograms, perfusion scans, and clinical updates) back to our institution. Preoperative and postoperative hemodynamics were compared using an exact 2-sided Wilcoxon rank sum test.

\section{RESULTS}

The first pediatric patient underwent PTE surgery at our institution in 1998. Since then 16 other patients, 18 years of age or younger, have successfully undergone PTE surgery by our surgeons $(n=17)$. This patient cohort constitutes $1.1 \%$ of the total number of operated CTEPH patients $(\mathrm{n}=1498)$ at UCSD between 1998 and 2009.

\section{Demographics}

Baseline patient data and clinical characteristics are presented in Table 1. The age range of the patients in this analysis was 8 to 18 years. There was a slight female predominance $(53 \%)$, and the majority were white $(76 \%)$.

The most common presenting symptom was exertional dyspnea and/or exercise intolerance $(94 \%)$. Slightly less than half had syncope or presyncope $(47 \%)$. Other presenting symptoms included chest pain (35\%), cough $(29 \%)$, cyanosis (18\%), edema (12\%), and hemoptysis (12\%). Only $35 \%$ of patients had known PE at the time of diagnosis, and up to $42 \%$ had a history or ultrasound findings consistent with a diagnosis of deep venous thrombosis. Idiopathic pulmonary arterial hypertension was the initial diagnosis in $29 \%$. A few patients were initially diagnosed with atypical pneumonia $(\mathrm{n}=3)$ or asthma $(\mathrm{n}=2)$. Hypoxemia requiring supplemental oxygen before surgery was present in $47 \%$ of patients. As part of their management, $65 \%$ underwent inferior vena caval filter placement before PTE surgery at UCSD.

\section{Thromboembolic Risk Factors}

Fifteen $(88 \%)$ of the 17 patients had 1 or more risk factors for thromboembolism as depicted in Tables 1 and 2. Fifteen of the 17 children had a hypercoagulable workup before PTE surgery with $67 \%$ of them testing positive for an underlying hypercoagulable state. The most common thrombophilic states were lupus anticoagulant $(\mathrm{n}=5)$, anticardiolipin antibody $(\mathrm{n}=4)$, and protein $\mathrm{C}$ deficiency $(\mathrm{n}=3)$, with 2 patients testing positive for both the lupus anticoagulant and anticardiolipin antibody. One patient exhibited testing suggestive of both protein $\mathrm{C}$ and $\mathrm{S}$ deficiency. Antithrombin III, factor V Leiden, and prothrombin gene defects were not seen in this cohort. A third of patients $(33 \%)$ had a positive family history of thromboembolism and/or hypercoagulable state.

Other disorders seen in this cohort that are associated with increased risk of thromboembolism ${ }^{12,13}$ include thoracic inlet obstruction $(\mathrm{n}=2)$, the presence of a ventriculoatrial shunt $(\mathrm{n}=1)$, nephrotic syndrome $(\mathrm{n}=1)$, and KlippelTrenaunay-Weber syndrome $(\mathrm{n}=1)$. Despite the young age of this cohort, 3 of 9 female patients were either on a regimen of contraceptive pills or were post partum at the time of diagnosis. Only 1 patient had a history of smoking. Another potential risk factor identified was prolonged immobilization of a limb, with $3(18 \%)$ patients having a prior fracture of their upper or lower limbs. However, the fracture did not appear to be related (temporally or locally) to the subsequent development of a deep venous thrombosis.

\section{Severity of Illness}

At presentation to UCSD, World Health Organization (WHO) functional status was assessed as follows: class I, $2(12 \%)$ of 17 patients; class II, 7 (41\%) of 17 patients; class III, $1(6 \%)$ of 17 patients; and class IV, $7(41 \%)$ of 17 patients. One of the patients in WHO class I status was deemed 
TABLE 1. Pediatric patient baseline demographics

\begin{tabular}{|c|c|c|c|c|c|c|c|c|}
\hline Patient & Age (y) & Gender & Race & Thrombosis risk & WHO score & Initial diagnosis & Oxygen & IVC filter \\
\hline 1 & 16 & $\mathrm{~F}$ & $\mathrm{~W}$ & $\mathrm{PC}$ & 3 & iPAH & $\mathrm{Y}$ & $\mathrm{N}$ \\
\hline 2 & 8 & $\mathrm{~F}$ & $\mathrm{~W}$ & & 4 & iPAH & $\mathrm{Y}$ & $\mathrm{N}$ \\
\hline 3 & 16 & M & AA & LA, ACLa & 2 & Lupus, DVT & $\mathrm{N}$ & $\mathrm{Y}$ \\
\hline 4 & 16 & $\mathrm{~F}$ & $\mathrm{~W}$ & TIO & 4 & $\mathrm{PE}$ & $\mathrm{Y}$ & $\mathrm{Y}(\mathrm{SVC})$ \\
\hline 5 & 15 & M & AA & Nephrotic syndrome & 2 & $\mathrm{PE}$ & $\mathrm{Y}$ & $\mathrm{Y}$ \\
\hline 6 & 13 & $\mathrm{~F}$ & $\mathrm{~W}$ & ACLa, increased FVIII & 4 & PE & $\mathrm{N}$ & $\mathrm{Y}$ \\
\hline 7 & 17 & M & $\mathrm{H}$ & $\mathrm{PC}, \mathrm{PS}$ & 2 & $\mathrm{iPAH}$ & $\mathrm{N}$ & $\mathrm{Y}$ \\
\hline 8 & 11 & M & $\mathrm{AA}$ & VA shunt & 2 & iPAH, VA thrombus & & $\mathrm{N}$ \\
\hline 9 & 16 & M & $\mathrm{W}$ & LA, ACLa & 1 & Hilar mass, AVM & $\mathrm{N}$ & $\mathrm{Y}$ \\
\hline 10 & 17 & $\mathrm{~F}$ & $\mathrm{~W}$ & $\mathrm{PC}$ & 4 & Asthma & $\mathrm{Y}$ & $\mathrm{Y}$ \\
\hline 11 & 13 & F & $\mathrm{W}$ & LA & 4 & iPAH & $\mathrm{Y}$ & $\mathrm{N}$ \\
\hline 12 & 15 & $\mathrm{~F}$ & $\mathrm{~W}$ & & 1 & $\mathrm{PE}$ & $\mathrm{N}$ & $\mathrm{Y}$ \\
\hline 13 & 17 & M & $\mathrm{W}$ & TIO & 2 & Atypical PNA & $\mathrm{Y}$ & $\mathrm{N}$ \\
\hline 14 & 17 & $\mathrm{~F}$ & $\mathrm{~W}$ & LA & 2 & "Saddle" PE & $\mathrm{N}$ & $\mathrm{Y}$ \\
\hline 15 & 10 & M & $\mathrm{W}$ & KTW & 4 & Asthma & $\mathrm{Y}$ & $\mathrm{Y}$ \\
\hline 16 & 15 & M & $\mathrm{W}$ & $\mathrm{ACLa}$ & 4 & PNA & $\mathrm{N}$ & $\mathrm{Y}$ \\
\hline 17 & 15 & $\mathrm{~F}$ & $\mathrm{~W}$ & LA, borderline ACLa & 2 & PNA & $\mathrm{N}$ & $\mathrm{N}$ \\
\hline
\end{tabular}

$W H O$, World Health Organization; $F$, female; $M$, male; $W$, white; $A A$, African American; $H$, Hispanic; $I V C$, inferior vena cava; $P C$, protein $C$ deficiency; $L A$, lupus anticoagulant; $A C L a$, anticardiolipin antibody; TIO, thoracic inlet obstruction; FVIII, factor VIII; PS, protein S deficiency; VA shunt, ventriculoatrial shunt; KTW, Klippel-Trenaunay-Weber syndrome; $i P A H$, idiopathic pulmonary arterial hypertension; $D V T$, deep venous thrombosis; $P E$, pulmonary embolus; $V A$, ventriculoatrial; $A V M$, arteriovenous malformation; $P N A$, pneumonia; $S V C$, superior vena cava.

appropriate for surgery owing to the presence of unilateral, main pulmonary artery chronic thromboembolic occlusive disease. The operation was performed in an effort to prevent the distal arteriopathy that can result from nonperfusion of an entire lung. ${ }^{14}$ The other WHO class I patient had significant collateral pulmonary circulation complicated by

TABLE 2. Thromboembolic risk factors

\begin{tabular}{lc}
\hline Deep venous thrombosis & $\mathrm{n}=4$ \\
Known & 3 \\
Suspected based on history & 5 \\
Thrombophilia & 4 \\
Lupus anticoagulant & 1 \\
Anticardiolipin antibody & 1 \\
Protein C deficiency & 0 \\
Protein S deficiency & 0 \\
Antithrombin III & 0 \\
Factor V Leiden & 1 \\
Prothrombin gene mutation & 1 \\
Elevated factor VIII & \\
Smoking & 2 \\
Other risk factors & 1 \\
Thoracic inlet obstruction & 1 \\
Ventriculoatrial shunt & 1 \\
Nephrotic kidney disease & \\
Klippel-Trenaunay-Weber & 0 \\
Splenectomy & 3 \\
Limb immobilization & \\
Female patients only & 2 \\
Oral contraceptive pill use & 1 \\
Peripartum & 5 \\
\hline Family history &
\end{tabular}

hemoptysis. PTE surgery was performed to potentially reverse and/or mitigate further vascular collateralization.

Baseline pulmonary hemodynamic measurements are shown in Table 3. For those patients with preoperative measurements, mean pulmonary arterial pressure was $45.5 \pm$ $20.7 \mathrm{~mm} \mathrm{Hg}$, mean cardiac output was $3.8 \pm 1.1 \mathrm{~L} / \mathrm{min}$, and pulmonary vasculature resistance (PVR) was $929 \pm$ 844 dynes $\cdot \mathrm{s} \cdot \mathrm{cm}^{-5}$. Preoperatively, $20 \%$ of our patients had a PVR greater than 1000 dynes $\cdot \mathrm{s} \cdot \mathrm{cm}^{-5}$, which has been associated with increased perioperative mortality. ${ }^{15}$

At presentation to UCSD for PTE surgery, $18 \%$ were on inotropic support: 1 patient was receiving milrinone and dobutamine, 1 was receiving milrinone and dopamine, and 1 patient was receiving dobutamine alone. Forty-one percent of patients were receiving pulmonary vasodilator therapy. Preoperative pulmonary hypertension-specific pharmacotherapy included epoprostenol $(\mathrm{n}=3)$, treprostinil $(n=1)$, bosentan $(n=3)$, sildenafil $(n=4)$, and/or inhaled nitric oxide $(\mathrm{n}=2)$.

\section{Surgical Results}

Mean cardiopulmonary bypass time was 219 minutes, mean right-sided circulatory arrest time 25 minutes, and mean left-sided circulatory arrest time 22 minutes. According to the Jamieson scoring system, $40 \%$ had type 1 disease, $40 \%$ type 2 , and $20 \%$ type 3 . No one had type 4 disease. A patent foramen ovale was closed in 4 patients $(24 \%)$ and the main pulmonary artery was enlarged with a pericardial patch in $2(12 \%)$ patients. Strikingly, $29 \%$ of patients had unilateral main pulmonary artery disease, with the right main pulmonary artery involved in $80 \%$ of these patients. 
TABLE 3. Cardiopulmonary hemodynamics before and after PTE surgery

\begin{tabular}{|c|c|c|c|c|c|c|c|c|c|}
\hline \multirow[b]{2}{*}{ Patient } & \multirow[b]{2}{*}{ Inotrope } & \multicolumn{4}{|c|}{ Preoperative } & \multicolumn{4}{|c|}{ Postoperative } \\
\hline & & $\begin{array}{c}\text { PAH } \\
\text { Rx } \\
\end{array}$ & $\begin{array}{c}\mathbf{P A P}(\mathbf{m}) \\
(\mathbf{m m} \mathbf{H g})\end{array}$ & $\begin{array}{c}\mathrm{CO} \\
(\mathrm{L} / \mathrm{min}) \\
\end{array}$ & $\begin{array}{c}\text { PVR } \\
\left(\text { dynes } \cdot \mathrm{s} \cdot \mathrm{cm}^{-5}\right) \\
\end{array}$ & PAH Rx & $\begin{array}{c}\mathbf{P A P}(\mathbf{m}) \\
(\mathbf{m m} \mathbf{H g})\end{array}$ & $\begin{array}{c}\text { CO } \\
(\mathrm{L} / \mathrm{min}) \\
\end{array}$ & $\begin{array}{c}\text { PVR } \\
\left(\text { dynes } \cdot \mathbf{s} \cdot \mathbf{c m}^{-5}\right) \\
\end{array}$ \\
\hline 1 & & & 63 & 4.21 & 969 & & 32 & 8.5 & 188 \\
\hline 2 & & E,B & 93 & 2 & 3240 & E & 66 & 3.6 & 1311 \\
\hline 3 & & & 40 & 4.1 & 624 & & 27 & 8.3 & 125 \\
\hline 4 & M, Dopa & E,B & 56 & 1.87 & 2053 & E,B & 31 & & \\
\hline 5 & & & 60 & 4.1 & 937 & & 38 & 4.4 & 473 \\
\hline 6 & Dobut & & & & & & 14 & 5.64 & 85 \\
\hline 7 & & & 31 & 4.51 & 337 & & 19 & 7.15 & 101 \\
\hline 8 & & S & & & 900 & & & & 370 \\
\hline 9 & & & 39 & 4.55 & 563 & & 24 & 5.59 & 157 \\
\hline 10 & & & 50 & 4.83 & 563 & & 25 & 4.6 & 304 \\
\hline 11 & & iNO & 57 & 2.2 & 1745 & & 25 & 3.6 & 422 \\
\hline 12 & & & 21 & 4.55 & 141 & & 19 & 4.1 & 117 \\
\hline 13 & & S,B & 45 & 3 & 880 & S,B & 37 & 6.54 & 355 \\
\hline 14 & & & 17 & 5.2 & 123 & & 17 & 4.73 & 152 \\
\hline 15 & M, Dobut & iNO,S,E,B & & & & $\mathrm{S}, \mathrm{B}$ & & & \\
\hline 16 & & $\mathrm{~S}, \mathrm{~T}$ & 48 & 4.1 & 741 & & 16 & 6.7 & 131 \\
\hline 17 & & & 17 & 4.27 & 112 & & 20 & 5.4 & 193 \\
\hline Mean \pm SD & & & $45.5 \pm 20.7$ & $3.8 \pm 1.1$ & $929 \pm 844$ & & $27.3 \pm 13.0^{*}$ & $5.6 \pm 1.6 \dagger$ & $299 \pm 307 \ddagger$ \\
\hline
\end{tabular}

$P T E$, Pulmonary thromboendarterectomy; $P A H R x$, treatment for pulmonary arterial hypertension; $P A P(m)$, pulmonary arterial pressure (mean); $C O$, cardiac output; $P V R$, pulmonary vascular resistance. Inotropes: $M$, Milirinone; Dopa, dopamine; Dobut, dobutamine. PAH Rx: E, epoprostenol; $B$, bosentan; $S$, sildenafil; $i N O$, inhaled nitric oxide; $T$, treprostinil. $* P<.001 . \dagger P<.01 . \ddagger P<.005$.

\section{Mortality}

No pediatric patient in this series died in surgery or within 30 days after surgery at our institution. Long-term survival (5+years) was $87.5 \%$. One pediatric death occurred in a patient with severe residual pulmonary hypertension (preoperative PVR, 3720 dynes $\cdot \mathrm{s} \cdot \mathrm{cm}^{-5}$; postoperative PVR, 1311 dynes $\cdot \mathrm{s} \cdot \mathrm{cm}^{-5}$ ) and the other patient stopped long-term anticoagulation and presented in pulseless electrical activity arrest after recurrent massive PE.

\section{Outcomes After PTE Surgery}

Median time to extubation was 1 day (range, $1-5$ days), median ICU stay 2 to 3 days (range, 2-11 days), and median hospital days 9 to 10 days (7-16 days). Fourteen patients had right heart catheterizations before and after PTE surgery; another 2 had catheterization data from either before or after surgery, but not both. There were dramatic improvements in hemodynamics after surgery as detailed in Table 3: mean pulmonary arterial pressure significantly decreased from $45.5 \pm 20.7 \mathrm{~mm} \mathrm{Hg}$ (range, $17-93 \mathrm{~mm} \mathrm{Hg}$ ) to $27.3 \pm 13.0 \mathrm{~mm} \mathrm{Hg}$ (range, $16-66 \mathrm{~mm} \mathrm{Hg})(P=.00073)$, PVR decreased from $929 \pm 844$ dynes $\cdot \mathrm{s} \cdot \mathrm{cm}^{-5}$ (range, 112-3420 dynes $\cdot \mathrm{s} \cdot \mathrm{cm}^{-5}$ ) to $299 \pm 307$ dynes $\cdot$ $\mathrm{s} \cdot \mathrm{cm}^{-5}\left(85-1311\right.$ dynes $\left.\cdot \mathrm{s} \cdot \mathrm{cm}^{-5}\right)(P=.0012)$ and cardiac output improved from $3.8 \pm 1.1 \mathrm{~L} / \mathrm{min}$ (range, $1.87-4.83 \mathrm{~L}$ / $\mathrm{min})$ to $5.6 \pm 1.6 \mathrm{~L} / \mathrm{min}$ (range, $3.8-8.5 \mathrm{~L} / \mathrm{min})(P=.0061)$. Only 1 patient had a PVR greater than 500 dynes $\cdot \mathrm{s} \cdot \mathrm{cm}^{-5}$ after surgery. None was discharged on inotropic or vasopressor support, and $24 \%$ were discharged on pulmonary vaso- dilators. Over half were free of cardiopulmonary symptoms (WHO class I) at follow-up, which ranges from 3 months to 7 years in this cohort.

There was, however, a significantly high rate of rethrombosis, occurring in up to $38 \%$ of patients having undergone PTE surgery $(n=6)$. This development occurred despite the fact that $59 \%$ of these patients were discharged with instructions to maintain an INR goal of 3 or more. Of those that had recurrent PE, 2 patients were known to have stopped their anticoagulation before rethrombosis, and 2 patients went on to require repeat PTE surgery.

\section{Postoperative Complications}

PTE surgery was generally well tolerated by all patients. Table 4 lists common postoperative complications and their occurrence in this population. Although reperfusion lung injury can occur in up to $40 \%$ of adult CTEPH patients, ${ }^{16,17}$ only 1 patient in this series had mild reperfusion lung injury requiring 2 extra days of supportive care in the ICU. No bleeding complications were noted in our patients despite the relatively aggressive postoperative anticoagulation and higher $(\geq 3)$ INR goals in the majority of patients. However, $5(29 \%)$ patients had moderatesevere pericardial effusions with 3 patients having tamponade physiology. The onset of these effusions, except one, occurred 2 to 3 weeks after discharge from the hospital. In 1 patient the effusion resolved with diuresis alone, 3 required steroids, 2 underwent pericardiocentesis, and 1 patient required a pericardial window. A relatively 
TABLE 4. Complications after PTE surgery

Rethrombosis

Hemorrhage requiring surgical exploration

Pneumothorax

Phrenic nerve injury

Recurrent laryngeal nerve injury

Wound infection

Moderate-severe pericardial effusion

Pericardial effusion with tamponade physiology

Pneumonia

Reperfusion lung injury

Atrial arrhythmias

Residual pulmonary hypertension

$\left(\mathrm{PVR}>500\right.$ dynes $\left.\cdot \mathrm{s} \cdot \mathrm{cm}^{-5}\right)$

On PAH pharmacotherapy at discharge

Delirium

Transient choreiform movements

$P T E$, Pulmonary thromboendarterectomy; $P V R$, pulmonary vascular resistance; $P A H$, pulmonary arterial hypertension; $C T E P H$, chronic thromboembolic pulmonary hypertension. *Complications seen in higher frequency in pediatric CTEPH patients than CTEPH patients in general. †Complications seen in lower frequency in pediatric CTEPH patients than CTEPH patients in general.

high incidence of postoperative delirium developed at a rate of $12 \%$ in this pediatric group, believed to be secondary to narcotic sensitivity in this opiod-naïve cohort.

\section{DISCUSSION}

This report details the largest case series of pediatric CTEPH patients who have successfully undergone PTE surgery, emphasizing not only that this operation is well tolerated, but also that it is associated with low morbidity, low mortality, and significant improvement in pulmonary hemodynamics and right heart function. This study also serves to highlight important features of pediatric CTEPH patients, including the increased presence of thrombophilic disorders, main pulmonary artery disease, and recurrent thrombosis risk.

Overall, the pediatric patient group makes up a small percentage $(1.1 \%)$ of the CTEPH patients who have undergone PTE surgery at our institution. CTEPH is generally underrecognized in the population at large, ${ }^{1}$ and this may be similarly true for pediatric patients. As is the case for adults, over half of the patients in this series did not have signs and symptoms indicative of acute embolism or deep venous thrombosis. Therefore, it is important for care providers to have a high index of suspicion for the possibility of pulmonary vascular disease in children who have exercise intolerance or exertional dyspnea. This is particularly true when a congenital heart condition has been ruled out and when the clinical picture does not support an alternative diagnosis such as asthma. There should be even more concern for the diagnosis if presyncope or syncope is present. Screening these children for CTEPH with ventilation-perfusion scanning is imperative inasmuch as a normal scan will completely rule out CTEPH. However, in patients with abnormal scans indicative of CTEPH, PTE surgery is largely curable and the natural history of this disease without surgery is extremely poor. ${ }^{5}$

In this study, $67 \%$ of the pediatric patients who had chronic thromboembolic disease exhibited an underlying thrombophilic disorder. This is in contrast to the adult CTEPH population, in which only $20 \%$ to $40 \%$ are reported to have a contributing hypercoagulable state. ${ }^{1,18,19}$ Lupus anticoagulant, anticardiolipin antibodies, and protein $\mathrm{C}$ deficiency were the most common predisposing thrombophilic states in this pediatric cohort. Even though antithrombin III, factor V Leiden, or prothrombin gene mutations were not detected, it does not exclude the possibility that children with these underlying disorders may also be at increased risk for CTEPH. In those without a hypercoagulable disorder, a different thromboembolic risk factor (ie, ventriculoarterial shunt, KlippelTrenaunay-Weber syndrome) was often present. Therefore, in children who have PE with an underlying hypercoagulable state and/or other thromboembolic risk factor, careful follow-up would be recommended to ensure complete resolution of the thrombus burden.

For selected patients with CTEPH, PTE surgery is the preferred treatment option given its established efficacy in improving cardiopulmonary hemodynamics, functional status, oxygenation, and survivorship. ${ }^{6,7,15-17,20-22}$ At baseline, most of the children were ill (WHO classes 2-4) and exhibited preoperative disease severity similar to adult CTEPH patients who have undergone PTE surgery. ${ }^{16,17,20-22}$ Over $40 \%$ of pediatric patients referred to UCSD were already receiving pharmacologic pulmonary arterial hypertension therapy, a rate similar to that currently seen in adult patients with operable CTEPH referred to UCSD for surgical consideration. ${ }^{23}$ This may reflect delayed recognition of CTEPH, a hesitancy to refer children for PTE surgery, an attempt to clinically stabilize those children with markedly elevated PVRs and right heart dysfunction, or simply reflect a growing trend to use medical therapies owing to the increased availability of pulmonary hypertension-specific medications. ${ }^{24}$

This study confirms that pediatric patients benefit dramatically from surgery and tolerate the operation well with significantly improved cardiopulmonary hemodynamics after PTE surgery. No deaths occurred in hospital or within 30 days of discharge at our institution. Long-term survival after PTE was $87.5 \%$, which is comparable with that of the CTEPH population at large $(75 \%-88.7 \%$ survival $) .{ }^{6,7}$ The majority of patients were asymptomatic at follow-up. Therefore, at medical centers experienced with PTE surgery and equipped to accommodate children, pediatric patients can have excellent outcomes from surgery. Because CTEPH is believed to lead to a progressive distal arteriopathy that is not responsive to surgery, ${ }^{4}$ prompt referral for PTE surgery 
should be considered for all CTEPH patients and young age should not be seen as an impediment to surgery.

Given the relatively small number of patients in this reported series, it is difficult to conclude that there are significant differences in postoperative complications when compared with the adult CTEPH patient. However, certain trends were observed. Reperfusion lung injury has been reported as a complication of PTE surgery $(16 \%-36 \%$ of surgically treated patients) and is a leading cause of mortality from this surgical procedure. ${ }^{16,17}$ In this series, only 1 patient had mild reperfusion lung injury that responded quickly (within 48 hours) to supportive measures. In addition, significant postoperative bleeding difficulties were rare. This was remarkable inasmuch as aggressive anticoagulation regimens were administered to almost $60 \%$ of the patients. Conversely, seen more frequently relative to older patients were pericardial fluid collections that required intervention. Five $(29 \%)$ of the pediatric patients had moderate to severe pericardial effusions after PTE, which is double the frequency $(3 \%-15 \%)$ seen in adult CTEPH patients. $^{16,17}$ Of these, 3 had cardiac tamponade and 4 of these episodes occurred 2 to 3 weeks after discharge from the hospital. It is not entirely clear why these patients might be at increased risk of having late, large pericardial effusions, although 3 of the 5 affected also had antiphospholipid antibodies. Whether an immune mechanism is important in the etiology of these effusions requires further investigation. What is evident, however, is that careful assessment is required for those pediatric patients who have an unexpected pain pattern, particularly in the chest, back, or shoulder region, several weeks after PTE surgery.

Despite the successful PTE operations in this cohort, there were 2 deaths that occurred during follow-up. One was from the sequelae of residual pulmonary hypertension and the other from rethrombosis when anticoagulation was stopped prematurely. Over a third of the patients $(38 \%)$ in this series went on to have new pulmonary thromboemboli as compared with only $1 \%$ to $4 \%$ of adult CTEPH patients. ${ }^{1,25}$ Factors potentially contributing to the development of postoperative thrombosis include the high rate of a thrombophilic state in the pediatric patient group, along with a higher incidence of unilateral, main pulmonary arterial disease $(29 \%$ vs $3 \%$ of adult CTEPH patients). The latter has been associated with a much higher risk of rethrombosis. ${ }^{14}$ This report also demonstrated that some of the pediatric patients who had repeat PE discontinued anticoagulation prematurely. This emphasizes the need for extensive, even repetitive, counseling of children with CTEPH regarding the importance of lifelong anticoagulation. It is not clear whether the use of inferior vena cava filters would benefit this population or decrease the risk of recurrent thrombosis, so no particular recommendation can be made in this regard. However, it is the practice at our in- stitution to aggressively anticoagulate any patient with main pulmonary artery disease or those with antiphospholipid antibodies with a continuous intravenous heparin infusion in the immediate postoperative period and to target higher INR goals (3.0-3.5 or 3.0-4.0) once there has been a transition to warfarin. In the setting of recurrent, surgical CTEPH, a second PTE may be feasible, as was the case for 2 patients in this series, and should be considered if clinically indicated.

\section{CONCLUSIONS}

This study demonstrates that pediatric CTEPH is rare, but amenable to PTE surgery, with excellent hemodynamic and survival outcomes. Patients should be carefully screened for underlying hypercoagulable states, and in those patients at highest risk for rethrombosis (ie, unilateral disease and positive anticardiolipin antibody), consideration should be given to placing an inferior vena cava filter and maintaining higher INR goals to reduce the likelihood of disease recurrence. All pediatric CTEPH patients deserve extensive counseling about the need for strict, lifelong adherence to anticoagulation. Inasmuch as there has been a dramatic increase in the recognition of venous thromboembolism in children over the past decade, special attention needs to be given to those whose emboli do not resolve. Referral for evaluation and possible PTE surgery should happen promptly to medical centers experienced and equipped to perform this surgery in children to minimize the distal vasculopathy that appears to occur over time in this disease.

We appreciate the collaboration with our biostatistician Aaron Kemp.

\section{References}

1. Auger WR, Fedullo PF. Chronic thromboembolic pulmonary hypertension. Sem Resp Crit Care Med. 2009;30:471-83 (review)

2. Pengo V, Lensing AW, Prins MH, Marchiori A, Davidson BL, Tiozzo F, et al. Thromboembolic Pulmonary Hypertension Study Group. Incidence of chronic thromboembolic pulmonary hypertension after pulmonary embolism. $N$ Engl J Med. 2004;350:2257-64.

3. Miniati M, Simonetta M, Bottai M, Scoscia E, Bauleo C, Tonelli L, et al. Survival and restoration of pulmonary perfusion in a long-term follow-up of patients after pulmonary embolism. Medicine. 2006;85:253-62

4. Moser K, Bloor C. Pulmonary vascular lesions occurring in patients with chronic major vessel thromboembolic pulmonary hypertension. Chest. 1993;103:685-92.

5. Reidel M, Stanek V, Widimsky, Prerovsky I. Longterm follow-up of patients with pulmonary embolism. Late prognosis and evolution of hemodynamic and respiratory data. Chest. 1982;81:151-8.

6. Archibald CJ, Auger PF, Fedullo PF, Channick RN, Kerr KM, Jamieson SW, et al. Long-term outcome after pulmonary thromboendarterectomy. Am J Respir Crit Care Med. 1999;160:523-8.

7. Corsico AG, D’Armini AM, Cerveri I, Klersy C, Ansaldo E, Niniano R, et al Long-term outcome after pulmonary endarterectomy. Am J Respir Crit Care Med. 2008;178:419-24

8. Raffini L, Huang YS, Witmer C, Feudtner C. Dramatic increase in venous thromboembolism in children's hospitals in the United States from 2001 to 2007. Pediatrics. 2009;124:1001-8.

9. Tormene D, Gavasso S, Rossetto, Simioni P. Thrombosis and thrombophilia in children: a systematic review. Semin Thromb Hemost. 2006;32:724-8. 
10. van Loon RLE, Roofhooft MTR, van Osch-Gevers $M$, Delhaas $T$, Strengers JL, Blom NA, et al. Clinical characterization of pediatric pulmonary hypertension: complex presentation and diagnosis. J Pediatr. 2009;155: 176-82.

11. Jamieson SW. Pulmonary thromboendarterectomy. In: Franco KL, Putnam JB, editors. Advanced therapy in thoracic surgery. Hamilton, Ontario: BC Decker; 1998. p. 310-8.

12. Gerotziafas GT. Risk factors for venous thromboembolism in children. Int Angiol. 2004;23:195-205.

13. Babyn PS, Gahunia HK, Massicotte P. Pulmonary thromboembolism in children. Pediatr Radiol. 2005;35:258-74.

14. Hirsch AM, Moser KM, Auger WR, Channick RN, Fedullo PF. Unilateral pulmonary artery thrombotic occlusion: is distal arteriopathy a consequence? Am J Respir Crit Care Med. 1996;154:491-6.

15. Jamieson SW, Kapelanski DP, Sakakibara N, Manecke GR, Thistlethwaite PA, Kerr KM, et al. Pulmonary endarterectomy: experience and lessons learned in 1,500 cases. Ann Thorac Surg. 2003;76:1457-62.

16. D'Armini A, Cattadori B, Monterosso C, Klersy C, Emmi V, Piovella F, et al. Pulmonary thromboendarterectomy in patients with chronic thromboembolic pulmonary hypertension: hemodynamic characteristic and changes. Eur J Cardiothorac Surg. 2000;18:696-702.

17. Thistlethwaite PA, Kaneko K, Madani MM, Jamieson SW. Technique and outcomes of pulmonary endarterectomy surgery. Ann Thorac Cardiovasc Surg. 2008; 14:274-82.
18. Wolf M, Boyer-Neumann C, Parent F, Jaillet H, Meyer D, et al. Thrombotic risk factors in pulmonary hypertension. Eur Respir J. 2000;15:395-9.

19. Bonderman D, Turecek PL, Jakowitsch J, Weltermann A, Adlbrecht C, Schneider B, et al. High prevalence of elevated clotting factor VIII in chronic thromboembolic pulmonary hypertension. Thromb Haemost. 2003;90:372-6.

20. Zoia MC, D'Armini AM, Beccaria M, Corsico A, Fulgoni P, Klersy C, et al. Mid term effects of pulmonary thromboendarterectomy on clinical and cardiopulmonary function status. Thorax. 2002;57:608-12.

21. Matsuda H, Ogino H, Minatoya K, Sasaki H, Nakanishi N, Kyotani S, et al. Longterm recovery of exercise ability after pulmonary endarterectomy for chronic thromboembolic pulmonary hypertension. Ann Thorac Surg. 2006;82:1338-43.

22. Freed DH, Thomson BM, Tsui SS, Dunning JJ, Sheares KK, Pepke-Zaba J, et al. Functional and haemodynamic outcome 1 year after pulmonary thromboendarterectomy. Eur J Cardiothorac Surg. 2008;34:525-30.

23. Jensen KW, Kerr KM, Fedullo PF, Kim NH, Test VJ, Ben-Yehuda O, et al. Pulmonary hypertensive medical therapy in chronic thromboembolic pulmonary hypertension before pulmonary thromboendarterectomy. Circulation. 2009;120: 1248-54.

24. Keogh AM, Mayer E, Benza RL, Corris P, Dartevelle PG, Frost AE, et al. Interventional and surgical modalities of treatment in pulmonary hypertension. $J$ Am Coll Cardiol. 2009;54:S67-77.

25. Ishida K, Masuda M, Tanaka H, Imamaki M, Katsumata M, Maruyama T, et al. Mid-term results for chronic thromboembolic pulmonary hypertension. Interact Cardiovasc Thorac Surg. 2009;9:626-9. 